\title{
SPECTRAL ANALYSIS FOR THE MATRIX STURM-LIOUVILLE OPERATOR ON A FINITE INTERVAL
}

\author{
N. BONDARENKO
}

\begin{abstract}
The inverse spectral problem is investigated for the matrix Sturm-Liouville equation on a finite interval. Properties of spectral characteristics are provided, a constructive procedure for the solution of the inverse problem along with necessary and sufficient conditions for its solvability is obtained.
\end{abstract}

\section{Introduction}

1.1. In this paper, the inverse spectral problem is investigated for the matrix SturmLiouville equation. Inverse spectral problems are to recover operators from their spectral characteristics.

The scalar case has been studied fairly completely (see $[1,2,3])$. The matrix case is more difficult for investigating. Different statements of inverse spectral problems for the matrix case were given in [4], [5] and [6] with corresponding uniqueness theorems. A constructive solution procedure was provided in [7], but for the special case of the simple spectrum only. Necessary and sufficient conditions were obtained in [8] for the case when the spectrum is asymptotically simple, that is an important restriction. Moreover, the method used by the authors of [8] does not give a reconstruction procedure. We also note that necessary and sufficient conditions on spectral data were given in [9] for Sturm-Liouville operators with matrixvalued potentials in the Sobolev space $W_{2}^{-1}$. This class of potentials differs from one considered in this paper.

In this paper, we study the self-adjoint matrix Sturm-Liouville operator in the general case, without any special restrictions on the spectrum. Properties of spectral characteristics are investigated, and necessary and sufficient conditions are obtained for the solvability of the inverse problem. We provide a constructive procedure for the solution of the inverse problem in the general case, that is a generalization of the algorithm from [7]. For solving the inverse problem we develop the ideas of the method of spectral mappings [3].

2000 Mathematics Subject Classification. 34A55 34B24 47E05.

Key words and phrases. Matrix Sturm-Liouville operators, inverse spectral problems, method of spectral mappings. 
1.2. Consider the boundary value problem $L=L(Q(x), h, H)$ for the matrix Sturm-Liouville equation:

$$
\begin{aligned}
\ell Y & :=-Y^{\prime \prime}+Q(x) Y=\lambda Y, \quad x \in(0, \pi), \\
U(Y) & :=Y^{\prime}(0)-h Y(0)=0, \quad V(Y):=Y^{\prime}(\pi)+H Y(\pi)=0 .
\end{aligned}
$$

Here $Y(x)=\left[y_{k}(x)\right]_{k=\overline{1, m}}$ is a column vector, $\lambda$ is the spectral parameter, and $Q(x)=$ $\left[Q_{j k}(x)\right]_{j, k=\overline{1, m}}$, where $Q_{j k}(x) \in L_{2}(0, \pi)$ are complex-valued functions. We will subsequently refer to the matrix $Q(x)$ as the potential. The boundary conditions are given by the matrices $h=\left[h_{j k}\right]_{j, k=\overline{1, m}}, H=\left[H_{j k}\right]_{j, k=\overline{1, m}}$, where $h_{j k}$ and $H_{j k}$ are complex numbers. In this paper we study the self-adjoint case, when $Q=Q^{*}, h=h^{*}, H=H^{*}$.

Let $\varphi(x, \lambda)$ and $S(x, \lambda)$ be matrix-solutions of equation (1) under the initial conditions

$$
\varphi(0, \lambda)=I_{m}, \quad \varphi^{\prime}(0, \lambda)=h, \quad S(0, \lambda)=0_{m}, \quad S^{\prime}(0, \lambda)=I_{m} .
$$

where $I_{m}$ is the identity $m \times m$ matrix, $0_{m}$ is the zero $m \times m$ matrix.

The function $\Delta(\lambda):=\operatorname{det}[V(\varphi)]$ is called the characteristic function of the boundary value problem $L$. The zeros of the entire function $\Delta(\lambda)$ coincide with the eigenvalues of $L$ (counting with their multiplicities, see Lemma 3), and they are real in the self-adjoint case.

Let $\omega=\omega^{*}$ be some $m \times m$ matrix. We will write $L(Q(x), h, H) \in A(\omega)$, if the problem $L$ has a potential from $L_{2}(0, \pi)$ and $h+H+\frac{1}{2} \int_{0}^{\pi} Q(x) d x=\omega$. Without loss of generality we may assume that $L \in A(\omega)$, where $\omega \in D=\left\{\omega: \omega=\operatorname{diag}\left\{\omega_{1}, \ldots, \omega_{m}\right\}, \omega_{1} \leq \ldots \leq \omega_{m}\right\}$.

One can achieve this condition applying the standard unitary transform.

In order to formulate the main result we need the following lemmas that will be proved in Section 2.

Lemma 1. Let $L \in A(\omega), \omega \in D$. The boundary value problem $L$ has a countable set of eigenvalues $\left\{\lambda_{n q}\right\}_{n \geq 0, q=\overline{1, m}}$, and

$$
\rho_{n q}=\sqrt{\lambda_{n q}}=n+\frac{\omega_{q}}{\pi n}+\frac{\kappa_{n q}}{n}, \quad\left\{\kappa_{n q}\right\}_{n \geq 0} \in l_{2}, q=\overline{1, m} .
$$

Let $\Phi(x, \lambda)=\left[\Phi_{j k}(x, \lambda)\right]_{j, k=\overline{1, m}}$ be a matrix-solution of equation (1) under the boundary conditions $U(\Phi)=I_{m}, V(\Phi)=0_{m}$. We call $\Phi(x, \lambda)$ the Weyl solution for $L$. Put $M(\lambda):=\Phi(0, \lambda)$. The matrix $M(\lambda)=\left[M_{j k}(\lambda)\right]_{j, k=\overline{1, m}}$ is called the Weyl matrix for $L$. The notion of the Weyl matrix is a generalization of the notion of the Weyl function ( $m$-function) for the scalar case (see [1], [3]). The Weyl functions and their generalizations often appear in applications and in pure mathematical problems, and they are natural spectral characteristics in the inverse problem theory for various classes of differential operators. 
Using the definition for $M(\lambda)$ one can easily check that

$$
M(\lambda)=-(V(\varphi))^{-1} V(S) .
$$

It follows from representation (4) that the matrix-function $M(\lambda)$ is meromorphic in $\lambda$ with simple poles in the eigenvalues $\left\{\lambda_{n q}\right\}$ of $L$ (see Lemma 4 ).

Denote

$$
\alpha_{n q}:=\underset{\lambda=\lambda_{n q}}{\operatorname{Res}} M(\lambda)
$$

The data $\Lambda:=\left\{\lambda_{n q}, \alpha_{n q}\right\}_{n \geq 0, q=\overline{1, m}}$ are called the spectral data of the problem $L$.

Let $\left\{\lambda_{n_{k} q_{k}}\right\}_{k \geq 0}$ be all the distinct eigenvalues from the collection $\left\{\lambda_{n q}\right\}_{n \geq 0, q=1, m}$. Put

$$
\alpha_{n_{k} q_{k}}^{\prime}:=\alpha_{n_{k} q_{k}}, k \geq 0, \quad \alpha_{n q}^{\prime}=0_{m},(n, q) \notin\left\{\left(n_{k}, q_{k}\right)\right\}_{k \geq 0} .
$$

Denote

$$
\begin{aligned}
1 & =m_{1}<\ldots<m_{p+1}=m+1, \\
\omega_{m_{s}} & =\ldots=\omega_{m_{s+1}-1}=: \omega^{(s)}, \quad s=\overline{1, p}
\end{aligned}
$$

where $p$ is the number of different values among $\left\{\omega_{q}\right\}_{q=\overline{1, m}}$. Let

$$
\alpha_{n}^{(s)}=\sum_{q=m_{s}}^{m_{s+1}-1} \alpha_{n q}^{\prime}, \quad s=\overline{1, p} .
$$

Lemma 2. Let $L \in A(\omega), \omega \in D$. Then the following relation holds

$$
\alpha_{n}^{(s)}=\frac{2}{\pi} I^{(s)}+\frac{\kappa_{n}^{(s)}}{n}, \quad\left\{\left\|\kappa_{n}^{(s)}\right\|\right\}_{n \geq 0} \in l_{2}, s=\overline{1, p},
$$

where

$$
I^{(s)}=\left[I_{j k}^{(s)}\right]_{j, k=\overline{1, m}}, \quad I_{j k}^{(s)}=\left\{\begin{array}{l}
1, m_{s} \leq j=k \leq m_{s+1}-1, \\
0, \quad \text { otherwise, }
\end{array}\right.
$$

and $\|$.$\| is a matrix norm: \|a\|=\max _{j, k} a_{j k}$.

Consider the following inverse problem.

Inverse Problem 1. Given the spectral data $\Lambda$, construct $Q, h$ and $H$.

We will write $\left\{\lambda_{n q}, \alpha_{n q}\right\}_{n \geq 0, q=\overline{1, m}} \in \mathrm{Sp}$, if for $\lambda_{n q}=\lambda_{k l}$ we always have $\alpha_{n q}=\alpha_{k l}$.

The main result of this paper is

Theorem 1. Let $\omega \in D$. For data $\left\{\lambda_{n q}, \alpha_{n q}\right\}_{n \geq 0, q=\overline{1, m}} \in S p$ to be the spectral data for a certain problem $L \in A(\omega)$ it is necessary and sufficient to satisfy the following conditions. 
(1) The asymptotics (3) and (5) are valid.

(2) All $\lambda_{n q}$ are real, $\alpha_{n q}=\left(\alpha_{n q}\right)^{*}, \alpha_{n q} \geq 0$ for all $n \geq 0, q=\overline{1, m}$, and the ranks of the matrices $\alpha_{n q}$ coincide with the multiplicities of $\lambda_{n q}$.

(3) For any row vector $\gamma(\lambda)$ that is entire in $\lambda$, and that satisfy the estimate

$$
\gamma(\lambda)=O(\exp (|\operatorname{Im} \sqrt{\lambda}| \pi)), \quad|\lambda| \rightarrow \infty
$$

if $\gamma\left(\lambda_{n q}\right) \alpha_{n q}=0$ for all $n \geq 0, q=\overline{1, m}$, then $\gamma(\lambda) \equiv 0$.

We prove necessity of the conditions of Theorem 1 in Section 2 and sufficiency in Section 4. In Section 3 the constructive procedure is provided for the solution of Inverse Problem 1.

\section{Necessity}

2.1. Let us study some properties of the spectral data.

Lemma 3. The zeroes of the characteristic function $\Delta(\lambda)$ coincide with the eigenvalues of the boundary value problem $L$. The multiplicity of each zero $\lambda_{0}$ of the function $\Delta(\lambda)$ equals to the multiplicity of the corresponding eigenvalue (by the multiplicity of the eigenvalue we mean the number of the corresponding linearly independent vector eigenfunctions).

Proof. (1) Let $\lambda_{0}$ be an eigenvalue of $L$, and let $Y^{0}$ be an eigenfunction corresponding to $\lambda_{0}$. Let us show that $Y^{0}(x)=\varphi\left(x, \lambda_{0}\right) Y^{0}(0)$. Clearly, $Y^{0}(0)=\varphi(0, \lambda) Y^{0}(0)$. It follows from $U\left(Y^{0}\right)=0$ that $Y^{0^{\prime}}(0)=h Y^{0}(0)=\varphi(0, \lambda) Y^{0}(0)$. Thus, $Y^{0}(x)$ and $\varphi\left(x, \lambda_{0}\right) Y^{0}(0)$ are the solutions for the same initial problem for the equation (1). Consequently, they are equal.

(2) Let us have exactly $k$ linearly independent eigenfunctions $Y^{1}, Y^{2}, \ldots, Y^{k}$ corresponding to the eigenvalue $\lambda_{0}$. Choose the invertible $m \times m$ matrix $C$ such that the first $k$ columns of $\varphi\left(x, \lambda_{0}\right) C$ coincide with the eigenfunctions. Consider $Y(x, \lambda):=\varphi(x, \lambda) C, Y(x, \lambda)=\left[Y_{q}(x\right.$,

$\lambda)]_{q=\overline{1, m}}, Y_{q}\left(x, \lambda_{0}\right)=Y^{q}(x), q=\overline{1, k}$. Clearly, that the zeros of $\Delta_{1}(\lambda):=\operatorname{det} V(Y)=\operatorname{det} V(\varphi)$. $\operatorname{det} C$ coincide with the zeros of $\Delta(\lambda)$ counting with their multiplicities. Note that $\lambda=\lambda_{0}$ is a zero of each of the columns $V\left(Y_{1}\right), \ldots, V\left(Y_{k}\right)$. Hence, if $\lambda_{0}$ is the zero of the determinants $\Delta_{1}(\lambda)$ and $\Delta(\lambda)$ with the multiplicity $p$, than $p \geq k$.

(3) Suppose that $p>k$. Rewrite $\Delta_{1}(\lambda)$ in the form

$$
\begin{gathered}
\Delta_{1}(\lambda)=\left(\lambda-\lambda_{0}\right)^{k} \Delta_{2}(\lambda) \\
\Delta_{2}(\lambda)=\operatorname{det}\left[\frac{V\left(Y_{1}\right)}{\lambda-\lambda_{0}}, \ldots, \frac{V\left(Y_{k}\right)}{\lambda-\lambda_{0}}, V\left(Y_{k+1}\right), \ldots, V\left(Y_{m}\right)\right] .
\end{gathered}
$$


In view our supposition, we have $\Delta_{2}\left(\lambda_{0}\right)=0$, i. e. there exist not all zero coefficients $\alpha_{q}, q=$ $\overline{1, m}$ zero exist such that

$$
\sum_{q=1}^{k} \alpha_{q} \frac{d V\left(Y_{q}\left(x, \lambda_{0}\right)\right)}{d \lambda}+\sum_{q=k+1}^{m} \alpha_{q} V\left(Y_{q}\left(x, \lambda_{0}\right)\right)=0 .
$$

If $\alpha_{q}=0$ for $q=\overline{1, k}$, then the function

$$
Y^{+}(x, \lambda):=\sum_{q=k+1}^{m} \alpha_{q} Y_{q}(x, \lambda)
$$

for $\lambda=\lambda_{0}$ is an eigenfunction corresponding to $\lambda_{0}$ that is linearly independent with $Y^{q}$, $q=\overline{1, k}$. Since the eigenvalue $\lambda_{0}$ has exactly $k$ corresponding eigenfunctions, we arrive at a contradiction.

Otherwise we consider the function

$$
Y^{+}(x, \lambda):=\sum_{q=1}^{k} \alpha_{q} Y_{q}(x, \lambda)+\left(\lambda-\lambda_{0}\right) \sum_{q=k+1}^{m} \alpha_{q} Y_{q}(x, \lambda) .
$$

It is easy to check that

$$
\begin{aligned}
\ell\left(Y^{+}(x, \lambda)\right) & =\lambda Y^{+}(x, \lambda), \quad \ell\left(\frac{d}{d \lambda} Y^{+}(x, \lambda)\right)=\lambda \frac{d}{d \lambda} Y^{+}(x, \lambda)+Y^{+}(x, \lambda), \\
U\left(Y^{+}\right) & =U\left(\frac{d}{d \lambda} Y^{+}\right)=0, \quad V\left(Y^{+}\left(x, \lambda_{0}\right)\right)=0 .
\end{aligned}
$$

Relation (6) is equivalent to the following one

$$
V\left(\frac{d}{d \lambda} Y^{+}\left(x, \lambda_{0}\right)\right)=0
$$

Thus, we obtain that $Y^{+}\left(x, \lambda_{0}\right)$ is an eigenfunction, and $\frac{d}{d \lambda} Y^{+}\left(x, \lambda_{0}\right)$ is a so-called associated function (see [10]) corresponding to $\lambda_{0}$. If we show that the considered Sturm-Liouville operator does not have associated functions, we will also arrive at a contradiction with $\Delta_{2}\left(\lambda_{0}\right) \neq 0$, and finally, prove that $k=p$.

(4) Let us prove that the self-adjoint operator given by (1), (2) does not have associated functions. Let $\lambda_{0}$ be an eigenvalue of $L$, and let $Y^{0}$ and $Y^{1}$ be a corresponding eigenfunction and an associated function respectively, i.e. both $Y^{0}$ and $Y^{1}$ satisfy (2) and

$$
\left(\ell-\lambda_{0}\right) Y^{0}=0, \quad\left(\ell-\lambda_{0}\right) Y^{1}=Y^{0} .
$$

This yields

$$
\left(\left(\ell-\lambda_{0}\right)^{2} Y^{1}, Y^{1}\right)=0
$$


for the scalar product defined by

$$
(Y, Z):=\int_{0}^{\pi} Y^{*}(x) Z(x) d x .
$$

In case of the self-adjoint operator, we have $(\ell Y, Z)=(Y, \ell Z)$ for any $Y$ and $Z$ satisfying (2), and the eigenvalue $\lambda_{0}$ is real. Therefore,

$$
\left(\left(\ell-\lambda_{0}\right) Y^{1},\left(\ell-\lambda_{0}\right) Y^{1}\right)=\left(Y^{0}, Y^{0}\right)=0
$$

and $Y^{0}=0$. Recall that $Y^{0}$ is the eigenfunction, and get a contradiction.

Lemma 4. All poles of the Weyl matrix $M(\lambda)$ are simple, and the ranks of the residue-matrices coincide with the multiplicities of the corresponding eigenvalues of $L$.

Proof. Let $\lambda_{0}$ be an eigenvalue of $L$ with a multiplicity $k$, and let $Y_{1}, Y_{2}, \ldots, Y_{k}$ be linearly independent vector eigenfunctions corresponding to $\lambda_{0}$. Following the proof of Lemma 3 , we introduce the invertible matrix $C=\left[C_{1}, \ldots, C_{m}\right]$ such that $Y_{q}(x)=\varphi\left(x, \lambda_{0}\right) C_{q}, q=\overline{1, k}$. Consider the vector-function $Y(x, \lambda)=\varphi(x, \lambda) C$. Clearly, that $(V(\varphi))^{-1}=C(V(Y))^{-1}$. Write $V(Y(x, \lambda))$ in the form

$$
V(Y(x, \lambda))=\left[\left(\lambda-\lambda_{0}\right) W_{1}(\lambda), \ldots,\left(\lambda-\lambda_{0}\right) W_{k}(\lambda), W_{k+1}(\lambda), \ldots, W_{m}(\lambda)\right]
$$

where

$$
\begin{aligned}
& W_{q}(\lambda)=\frac{V\left(Y_{q}(x, \lambda)\right)}{\lambda-\lambda_{0}}, \quad q=\overline{1, k} \\
& W_{q}(\lambda)=V\left(Y_{q}(\lambda)\right), \quad q=\overline{k+1, m} .
\end{aligned}
$$

Clearly, $W_{q}(\lambda)$ are entire functions, and

$$
\operatorname{det} W(\lambda)=\operatorname{det}\left[W_{1}(\lambda), \ldots, W_{m}(\lambda)\right] \neq 0
$$

for $\lambda$ from a sufficiently small neighborhood of $\lambda_{0}$ (otherwise the multiplicity of the eigenvalue $\lambda_{0}$ is greater than $k$ ). It is easy to show that

$$
\begin{aligned}
& \operatorname{det} V(Y(x, \lambda))=\left(\lambda-\lambda_{0}\right)^{k} \operatorname{det} W(\lambda), \\
& (V(Y(x, \lambda)))^{-1}=\left[\frac{X_{1}(\lambda)}{\lambda-\lambda_{0}}, \ldots, \frac{X_{k}(\lambda)}{\lambda-\lambda_{0}}, X_{k+1}(\lambda), \ldots, X_{m}(\lambda)\right]^{t},
\end{aligned}
$$

where $X_{q}(\lambda)$ are analytic in a sufficiently small neighborhood of $\lambda_{0}$ (the superscript $t$ stands for transposition). Using (4) we get

$$
\alpha_{0}=\operatorname{Res}_{\lambda=\lambda_{0}} M(\lambda)=-\operatorname{Res}_{\lambda=\lambda_{0}}(V(\varphi(x, \lambda)))^{-1} V(S(x, \lambda))
$$




$$
\begin{aligned}
& =-\operatorname{Res}_{\lambda=\lambda_{0}}\left[\frac{X_{1}(\lambda)}{\lambda-\lambda_{0}}, \ldots, \frac{X_{k}(\lambda)}{\lambda-\lambda_{0}}, X_{k+1}(\lambda), \ldots, X_{m}(\lambda)\right]^{t} V(S(x, \lambda)) \\
& =-C\left[X_{1}\left(\lambda_{0}\right), \ldots, X_{k}\left(\lambda_{0}\right), 0, \ldots, 0\right]^{t} V\left(S\left(x, \lambda_{0}\right)\right)=-X V\left(S\left(x, \lambda_{0}\right)\right) .
\end{aligned}
$$

Therefore, the poles of the Weyl matrix are simple, and $\operatorname{rank} \alpha_{0} \leq k$.

Let us prove the reverse inequality. Note that

$$
\operatorname{Res}_{\lambda=\lambda_{0}}(V(\varphi(x, \lambda)))^{-1} V(\varphi(x, \lambda))=0_{m}=X V\left(\varphi\left(x, \lambda_{0}\right)\right) .
$$

Let $\psi\left(x, \lambda_{0}\right)$ be a solution of equation (1) for $\lambda=\lambda_{0}$ under the initial condition $V(\psi)=X^{*}$. Since columns of the matrices $\varphi\left(x, \lambda_{0}\right)$ and $S\left(x, \lambda_{0}\right)$ form a fundamental system of solutions of equation (1), we have

$$
\begin{aligned}
\psi\left(x, \lambda_{0}\right) & =\varphi\left(x, \lambda_{0}\right) A+S\left(x, \lambda_{0}\right) B, \\
X X^{*} & =X V\left(\psi\left(x, \lambda_{0}\right)\right)=X V\left(\varphi\left(x, \lambda_{0}\right)\right) A+X V\left(S\left(x, \lambda_{0}\right)\right) B=-\alpha_{0} B .
\end{aligned}
$$

On the one hand, since $\operatorname{det} W \neq 0$, the vectors $X_{q}\left(\lambda_{0}\right)$ are linearly independent, therefore, $\operatorname{rank} X X^{*}=k$. On the other hand, $r a n k \alpha_{0} B \leq r a n k \alpha_{0}$. Thus, we conclude that $r a n k \alpha_{0} \geq k$.

Lemma 5. Let $\lambda_{0}, \lambda_{1}$ be eigenvalues of $L, \lambda_{0} \neq \lambda_{1}$, and $\alpha_{i}=\underset{\lambda=\lambda_{i}}{\operatorname{Res}} M(\lambda), i=0,1$. The following relations hold

$$
\begin{aligned}
& \alpha_{0}^{*} \int_{0}^{\pi} \varphi^{*}\left(x, \lambda_{0}\right) \varphi\left(x, \lambda_{0}\right) d x \alpha_{0}=\alpha_{0}^{*}, \\
& \alpha_{0}^{*} \int_{0}^{\pi} \varphi^{*}\left(x, \lambda_{0}\right) \varphi\left(x, \lambda_{1}\right) d x \alpha_{1}=0_{m} .
\end{aligned}
$$

In particular, according to the first relation,

$$
\alpha_{0}=\alpha_{0}^{*} \geq 0
$$

Proof. Denote

$$
\ell^{*} Z:=-Z^{\prime \prime}+Z Q(x), \quad V^{*}(Z):=Z^{\prime}(\pi)+Z(\pi) H, \quad\langle Z, Y\rangle:=Z^{\prime} Y-Z Y^{\prime},
$$

where $Z=\left[Z_{k}\right]_{k=\overline{1, m}}^{t}$ is a row vector ( $t$ is the sign for the transposition). Then

$$
\langle Z, Y\rangle_{x=\pi}=V^{*}(Z) Y(\pi)-Z(\pi) V(Y) .
$$

If $Y(x, \lambda)$ and $Z(x, \mu)$ satisfy the equations $\ell Y(x, \lambda)=\lambda Y(x, \lambda)$ and $\ell^{*} Z(x, \mu)=\mu Z(x, \mu)$, respectively, then $\frac{d}{d x}\langle Z, Y\rangle=(\lambda-\mu) Z Y$. In particular, if $\lambda=\mu$, then $\langle Z, Y\rangle$ does not depend on $x$. 
Since $\lambda_{0}$ is real, $\varphi^{*}\left(x, \lambda_{0}\right)$ satisfies the equation $\ell^{*} Z=\lambda_{0} Z$. Hence,

$$
\begin{aligned}
\int_{0}^{\pi} \varphi^{*}\left(x, \lambda_{0}\right) \varphi\left(x, \lambda_{0}\right) d x & =\lim _{\lambda \rightarrow \lambda_{0}} \frac{\left.\left\langle\varphi^{*}\left(x, \lambda_{0}\right), \varphi(x, \lambda)\right\rangle\right|_{0} ^{\pi}}{\lambda-\lambda_{0}} \\
& =\lim _{\lambda \rightarrow \lambda_{0}} \frac{V^{*}\left(\varphi^{*}\left(x, \lambda_{0}\right)\right) \varphi(x, \lambda)-\varphi^{*}\left(x, \lambda_{0}\right) V(\varphi(x, \lambda))}{\lambda-\lambda_{0}} .
\end{aligned}
$$

It follows from (4) and Lemma 4 that

$$
V\left(\varphi\left(x, \lambda_{0}\right)\right) \alpha_{0}=-\lim _{\lambda \rightarrow \lambda_{0}}\left(\lambda-\lambda_{0}\right) V(\varphi(x, \lambda))(V(\varphi(x, \lambda)))^{-1} V(S(x, \lambda))=0_{m} .
$$

Analogously $\alpha_{0}^{*} V^{*}\left(\varphi^{*}\left(x, \lambda_{0}\right)\right)=0_{m}$. Consequently, we calculate

$$
\begin{aligned}
\alpha_{0}^{*} \int_{0}^{\pi} \varphi^{*}\left(x, \lambda_{0}\right) \varphi\left(x, \lambda_{0}\right) d x \alpha_{0} & =\alpha_{0}^{*} \varphi^{*}\left(\pi, \lambda_{0}\right) \lim _{\lambda \rightarrow \lambda_{0}} \frac{V(\varphi(x, \lambda))}{\lambda-\lambda_{0}} \\
\times \lim _{\lambda \rightarrow \lambda_{0}}\left(\lambda-\lambda_{0}\right)(V(\varphi(x, \lambda)))^{-1} V(S(x, \lambda)) & =\alpha_{0}^{*} \varphi^{*}\left(\pi, \lambda_{0}\right) V\left(S\left(x, \lambda_{0}\right)\right) \\
& =-\alpha_{0}^{*}\left\langle\varphi^{*}\left(x, \lambda_{0}\right), S\left(x, \lambda_{0}\right)\right\rangle_{x=\pi} \\
& =-\alpha_{0}^{*}\left\langle\varphi^{*}\left(x, \lambda_{0}\right), S\left(x, \lambda_{0}\right)\right\rangle_{x=0}=\alpha_{0}^{*} .
\end{aligned}
$$

Similarly one can derive the second relation of the lemma.

2.2. In this subsection we obtain asymptotics for the spectral data.

Denote $\rho:=\sqrt{\lambda}, \operatorname{Re} \rho \geq 0, \tau:=\operatorname{Im} \rho, G_{\delta}=\{\rho:|\rho-k| \geq \delta, k=0,1,2, \ldots\}, \delta>0$. By the standard way (see [3, Sec. 1.1]) one can obtain the estimate

$$
\begin{aligned}
\Delta(\lambda)= & (-\rho \sin \rho \pi)^{m}+O\left(|\rho|^{m-1} \exp (m|\tau| \pi)\right)=(-\rho \sin \rho \pi)^{m}+(-\rho \sin \rho \pi)^{m-1} O(\exp (|\tau| \pi)) \\
& +\ldots+(-\rho \sin \rho \pi) O(\exp ((m-1)|\tau| \pi))+O(\exp (m|\tau| \pi)), \quad|\rho| \rightarrow \infty .
\end{aligned}
$$

Proof of Lemma 1. 1) Consider the contour $\Gamma_{N}=\left\{\lambda:|\lambda|=(N+1 / 2)^{2}\right\}$. By virtue of (7)

$$
\Delta(\lambda)=f(\lambda)+g(\lambda), \quad f(\lambda)=(-\rho \sin \rho \pi)^{m}, \quad|g(\lambda)| \leq C|\rho|^{m-1} \exp (m|\tau| \pi) .
$$

If $\lambda \in \Gamma_{N}$ for sufficiently large $N$, we have $|f(\lambda)|>|g(\lambda)|$. Then by Rouche's theorem the number of zeros of $\Delta(\lambda)$ inside $\Gamma_{N}$ coincide with the number of zeros of $f(\lambda)$ (counting with their multiplicities), i.e. it equals $(N+1) m$. Thus, in the circle $|\lambda|<(N+1 / 2)^{2}$ there are exactly $(N+1) m$ eigenvalues of $L:\left\{\lambda_{n q}\right\}_{n=\overline{0, N}, q=\overline{1, m}}$.

Applying Rouche's theorem to the circle $\gamma_{n}(\delta)=\{\rho:|\rho-n| \leq \delta\}$, we conclude that for sufficiently large $n$ there are exactly $m$ zeros of $\Delta\left(\rho^{2}\right)$ lying inside $\gamma_{n}(\delta)$, namely $\left\{\rho_{n q}\right\}_{q=\overline{1, m}}$. Since $\delta>0$ is arbitrary, it follows that

$$
\rho_{n q}=n+\varepsilon_{n q}, \quad \varepsilon_{n q}=o(1), \quad n \rightarrow \infty .
$$


Using (7) for $\rho=\rho_{n q}$, we get

$$
\left(-\rho_{n q} \sin \rho_{n q} \pi\right)^{m}+\left(-\rho_{n q} \sin \rho_{n q} \pi\right)^{m-1} O(1)+\ldots+\left(-\rho_{n q} \sin \rho_{n q} \pi\right) O(1)+O(1)=0, \quad n \rightarrow \infty .
$$

Denote $s_{n q}:=\left|\rho_{n q} \sin \rho_{n q} \pi\right|$, and rewrite the obtained estimate in the form

$$
s_{n q}^{m} \leq C_{0}+C_{1} s_{n q}+\ldots+C_{m-1} s_{n q}^{m-1} .
$$

It follows from (9) that $s_{n q} \leq \max \left\{1, \sum_{k=0}^{m-1} C_{k}\right\}$. Otherwise we arrive at a contradiction:

$$
s_{n q}^{m}>\sum_{k=0}^{m-1} C_{k} s_{n q}^{m-1} \geq \sum_{k=0}^{m-1} C_{k} s_{n q}^{k} .
$$

Hence, $\left|\rho_{n q} \sin \rho_{n q} \pi\right| \leq C$. Using (8) we get

$$
\sin \rho_{n q} \pi=\sin \varepsilon_{n q} \pi \cos n \pi=O\left(n^{-1}\right), \quad \varepsilon_{n q}=O\left(n^{-1}\right), \quad n \rightarrow \infty .
$$

Together with (8) this yields

$$
\rho_{n q}=n+O\left(n^{-1}\right), \quad n \rightarrow \infty .
$$

2) Let us derive the more precise asymptotic formula. One can easily show that

$$
V(\varphi)=-\rho \sin \rho \pi \cdot I_{m}+\omega \cos \rho \pi+\kappa(\rho),
$$

where

$$
\kappa(\rho)=\frac{1}{2} \int_{0}^{\pi} Q(t) \cos \rho(\pi-2 t) d t+O\left(\frac{1}{\rho} \exp (|\tau| \pi)\right) .
$$

Consider the linear mappings $z_{n}(\rho)$ that map the circles $\{\rho:|\rho-n| \leq C / n\}$ (note that $\rho_{n q}$ lie in these circles for a fixed sufficiently large $C$ ) to the circle $\{z:|z| \leq R\}$ :

$$
\rho=n+\frac{z_{n}(\rho)}{\pi n} .
$$

For $|z| \leq R$ we have

$$
V(\varphi)=(-1)^{n}\left(\omega-z_{n}(\rho) I_{m}+\kappa_{n}\left(z_{n}(\rho)\right)\right) .
$$

Using the representation for $\kappa(\rho)$ we get $\kappa_{n}(z)=o(1), n \rightarrow \infty$, uniformly with respect to $z$ in the circle $\{z:|z| \leq R\}$. Moreover, for each sequence $\left\{z_{n}^{0}\right\}_{n \geq 0} \subset\{z:|z| \leq R\}$ we have $\left\{\left\|\kappa_{n}\left(z_{n}^{0}\right)\right\|\right\}_{n \geq 0} \in$ $l_{2}$ and $\sum_{n \geq 0}\left\|\kappa_{n}\left(z_{n}^{0}\right)\right\|^{2}<C$, where $C$ is some constant. Consequently,

$$
\Delta\left(\rho^{2}\right)= \pm f\left(z_{n}(\rho)\right)+g_{n}\left(z_{n}(\rho)\right)
$$

where $f(z)=\operatorname{det}\left(\omega-z I_{m}\right), g_{n}(z)=o(1), n \rightarrow \infty$ (uniformly with respect to $z \in\{z:|z| \leq R\}$ ), and the choice of sign \pm depends only on $n$. Fix $0<\delta<1 / 2 \min _{q, l: \omega_{q} \neq \omega_{l}}\left|\omega_{q}-\omega_{l}\right|$ and introduce 
the contours $\gamma_{q}=\left\{z:\left|z-\omega_{q}\right|=\delta\right\}$. Clearly, the inequality $|f(z)|>\left|g_{n}(z)\right|$ holds on $\gamma_{q}$ for all sufficiently large $n$, and by Rouche's theorem two analytic functions $\Delta\left(\rho_{n}^{2}(z)\right)$ and $f(z)$ have an equal number of zeros inside $\gamma_{q}$ (here $\rho_{n}$ is the inverse mapping to $z_{n}$ ). Thus, we have

$$
\rho_{n q}=n+\frac{\omega_{q}}{\pi n}+\frac{\kappa_{n q}}{n}, \quad \kappa_{n q}=o(1), n \rightarrow \infty, \quad q=\overline{1, m} .
$$

Substituting this formula into (10) we get

$$
V(\varphi)=(-1)^{n}\left(\omega-\omega_{q} I_{m}-\pi \kappa_{n q} I_{m}+\kappa_{n}\left(z_{n}\left(\rho_{n q}\right)\right)\right)
$$

Since $\left\{\left\|\kappa_{n}\left(z_{n}\left(\rho_{n q}\right)\right)\right\|\right\} \in l_{2}$, one can easily prove that $\left\{\kappa_{n q}\right\} \in l_{2}$.

Proof of Lemma 2. 1) Let $\tilde{M}(\lambda)$ be the Weyl matrix for the problem $\tilde{L}(\tilde{Q}, \tilde{h}, \tilde{H})$, such that $\tilde{Q}(x)=$ $\frac{2}{\pi} \omega, \tilde{h}=\tilde{H}=0_{m}$. Then $\tilde{\alpha}^{(s)}=\frac{2}{\pi} I^{(s)}, s=\overline{1, p}$.

Consider the contours $\gamma_{n}^{(s)}=\left\{\lambda:\left|\lambda-\left(n^{2}+\frac{2}{\pi} \omega^{(s)}\right)\right|=R\right\}, R=\frac{1}{\pi} \min _{q, l: \omega_{q} \neq \omega_{l}}\left|\omega_{q}-\omega_{l}\right|$. Using the residue theorem and taking Lemma 1 into account, we deduce

$$
\frac{1}{2 \pi i} \int_{\gamma_{n}^{(s)}}(M(\lambda)-\tilde{M}(\lambda)) d \lambda=\sum_{q=m_{s}}^{m_{s+1}-1} \alpha_{n q}^{\prime}-\sum_{q=m_{s}}^{m_{s+1}-1} \tilde{\alpha}_{n q}^{\prime}=\alpha_{n}^{(s)}-\frac{2}{\pi} I^{(s)}, n \geq n^{*}, s=\overline{1, p} .
$$

One can easily show that $M_{j k}(\lambda)=-\frac{\Delta_{j k}(\lambda)}{\Delta(\lambda)}$, where

$$
\Delta_{j k}(\lambda)=\operatorname{det}\left[V\left(\varphi_{1}\right), \ldots, V\left(\varphi_{j-1}\right), V\left(S_{k}\right), V\left(\varphi_{j+1}\right), \ldots, V\left(\varphi_{m}\right)\right]
$$

Using this representation, we arrive at

$$
M_{j k}(\lambda)-\tilde{M}_{j k}(\lambda)=\frac{\Delta(\lambda) \tilde{\Delta}_{j k}(\lambda)-\Delta_{j k}(\lambda) \tilde{\Delta}(\lambda)}{\Delta(\lambda) \tilde{\Delta}(\lambda)}, \quad j, k=\overline{1, m} .
$$

Let us use the mappings $z_{n}$ introduced in the proof of Lemma 1:

$$
\rho=n+\frac{z_{n}(\rho)}{\pi n}
$$

If $\lambda \in \gamma_{n}^{(s)}$, then $0<\delta_{1} \leq\left|z_{n}(\rho)-\omega_{q}\right|$ for all $q=\overline{1, m}$, and $\left|z_{n}(\rho)-\omega^{(s)}\right| \leq \delta_{2}$. Hence, the estimate for $\Delta(\lambda)$ obtained in the proof of Lemma 1 is valid: $\Delta(\lambda)= \pm f\left(z_{n}(\rho)\right)+o(1), \lambda \in \gamma_{n}^{(s)}, n \rightarrow \infty$ (uniformly with respect to $\lambda$ ).

Similarly, we estimate

$$
\begin{aligned}
& \Delta_{j k}(\lambda)= \pm \frac{f\left(z_{n}(\rho)\right)}{z_{n}(\rho)-\omega_{j}}+o(1) \quad \text { for } j=k, \\
& \Delta_{j k}(\lambda)=o(1) \quad \text { for } j \neq k, \lambda \in \gamma_{n}^{(s)}, n \rightarrow \infty, \quad j, k=\overline{1, m} .
\end{aligned}
$$


Convergence of the remainders is uniform with respect to $\lambda$, the choice of sign \pm depends only on $n$. Analogous relations hold for $\tilde{\Delta}(\lambda)$ and $\tilde{\Delta}_{j k}(\lambda)$.

Substituting these estimates into (11) and taking into account that $C_{1} \leq\left|f\left(z_{n}(\rho)\right)\right| \leq C_{2}$ for $\lambda \in \gamma_{n}^{(s)}$, we arrive at

$$
\begin{aligned}
M_{j k}(\lambda)-\tilde{M}_{j k}(\lambda) & =o(1), \quad j, k=\overline{1, m}, \quad \lambda \in \gamma_{n}^{(s)}, \\
\frac{1}{2 \pi i} \int_{\gamma_{n}^{(s)}}(M(\lambda)-\tilde{M}(\lambda)) d \lambda & =o(1), \quad \alpha_{n}^{(s)}=\frac{2}{\pi} I^{(s)}+\eta_{n}^{(s)}, \eta_{n}^{(s)}=o(1), \quad n \rightarrow \infty .
\end{aligned}
$$

(2) Below one and the same symbol $\left\{\kappa_{n}\right\}$ denotes various matrix sequences such that $\left\{\left\|\kappa_{n}\right\|\right\} \in l_{2}$. Using the standard asymptotics

$$
\begin{aligned}
\varphi(x, \lambda)= & \cos \rho x \cdot I_{m}+Q_{1}(x) \frac{\sin \rho x}{\rho}+\int_{0}^{x} \frac{\sin \rho(x-2 t)}{2 \rho} Q(t) d t+O\left(\frac{\exp |\tau| x}{\rho}\right), \\
& |\rho| \rightarrow \infty, \quad x \in[0, \pi],
\end{aligned}
$$

where $Q_{1}(x)=h+\int_{0}^{x} Q(t) d t$, one can easily show that

$$
\int_{0}^{\pi} \varphi^{*}\left(x, \lambda_{n q}\right) \varphi\left(x, \lambda_{n l}\right) d x=\frac{\pi}{2} I_{m}+\frac{\kappa_{n}}{n}, \quad \lambda_{n q}-\lambda_{n l}=\frac{\kappa_{n}}{n} .
$$

Applying Lemma 5, we get

$$
\alpha_{n q}\left(\frac{\pi}{2} I_{m}+\frac{\kappa_{n}}{n}\right) \alpha_{n q}=\alpha_{n q}, \quad n \geq 0, \quad q=\overline{1, m} .
$$

Clearly, $\left\|\alpha_{n q}\right\| \leq C, n \geq 0, q=\overline{1, m}$. Consequently, $\frac{\pi}{2} \alpha_{n q}^{2}=\alpha_{n q}+\frac{\kappa_{n}}{n}$. Similarly we derive $\alpha_{n q} \alpha_{n l}=\frac{\kappa_{n}}{n}, m_{s} \leq q, l \leq m_{s+1}-1, q \neq l, s=\overline{1, p}$. Thus,

$$
\frac{\pi}{2}\left(\alpha_{n}^{(s)}\right)^{2}=\frac{\pi}{2}\left(\sum_{q=m_{s}}^{m_{s+1}-1} \alpha_{n q}^{\prime}\right)^{2}=\frac{\pi}{2} \sum_{q=m_{s}}^{m_{s+1}-1}\left(\alpha_{n q}^{\prime}\right)^{2}+\frac{\kappa_{n}}{n}=\sum_{q=m_{s}}^{m_{s+1}-1} \alpha_{n q}^{\prime}+\frac{\kappa_{n}}{n}=\alpha_{n}^{(s)}+\frac{\kappa_{n}}{n} .
$$

Substitute the result of point 1 into this equality:

$$
\begin{aligned}
\frac{\pi}{2}\left(\frac{2}{\pi} I^{(s)}+\eta_{n}^{(s)}\right)^{2} & =\frac{2}{\pi} I^{(s)}+\eta_{n}^{(s)}+\frac{\kappa_{n}}{n}, \\
\left(I_{m}-2 I^{(s)}\right) \eta_{n}^{(s)} & =\frac{\pi}{2}\left(\eta_{n}^{(s)}\right)^{2}+\frac{\kappa_{n}}{n} .
\end{aligned}
$$

Consequently, $\eta_{n}^{(s)}=\frac{\kappa_{n}}{n}$.

2.3. Proof of Theorem 1 (necessity). The first two conditions are fulfilled by Lemmas 1, 2, 4, 5 .

Let $\gamma(\lambda)$ be a function described in condition 3. Recall that

$$
V\left(\varphi\left(x, \lambda_{n q}\right)\right) \alpha_{n q}=0_{m} .
$$


Since

$$
\operatorname{rank} V\left(\varphi\left(x, \lambda_{n q}\right)\right)+\operatorname{rank} \alpha_{n q}=m
$$

and $\gamma\left(\lambda_{n q}\right) \alpha_{n q}=0$, we get $\gamma\left(\lambda_{n q}\right)=C_{n q} V\left(\varphi\left(x, \lambda_{n q}\right)\right)$, i. e. the row $\gamma\left(\lambda_{n q}\right)$ is a linear combination of the rows of the matrix $V\left(\varphi\left(x, \lambda_{n q}\right)\right.$ (here $C_{n q}$ is a row of coefficients). Consider

$$
f(\lambda)=\gamma(\lambda)(V(\varphi(x, \lambda)))^{-1} .
$$

The matrix-function $(V(\varphi(x, \lambda)))^{-1}$ has simple poles in $\lambda=\lambda_{n q}$, therefore, we calculate

$$
\begin{aligned}
\operatorname{Res}_{\lambda=\lambda_{n q}} f(\lambda) & =\gamma\left(\lambda_{n q}\right) \operatorname{Res}_{\lambda=\lambda_{n q}}(V(\varphi(x, \lambda)))^{-1} \\
& =C_{n q} \lim _{\lambda \rightarrow \lambda_{n q}} V(\varphi(x, \lambda)) \lim _{\lambda \rightarrow \lambda_{n q}}\left(\lambda-\lambda_{n q}\right)(V(\varphi(x, \lambda)))^{-1}=0 .
\end{aligned}
$$

Hence, $f(\lambda)$ is entire. It is easy to show that

$$
\left\|(V(\varphi(x, \lambda)))^{-1}\right\| \leq C_{\delta}|\rho|^{-1} \exp (-|\tau| \pi), \quad \rho \in G_{\delta},
$$

where $G_{\delta}=\{\rho:|\rho-k| \geq \delta, k=0,1,2, \ldots\}, \delta>0$. From this we conclude that $\|f(\lambda)\| \leq \frac{C}{|\rho|}$ in $G_{\delta}$. By the maximum principle this estimate is valid in the whole $\lambda$-plane. Using Liouville's theorem, we obtain $f(\lambda) \equiv 0$. Consequently, $\gamma(\lambda) \equiv 0$.

Note that in the scalar case condition 3 follows from the first two conditions of Theorem 1. Indeed, in the scalar case, we have $\gamma\left(\lambda_{n}\right) \alpha_{n}=0, n \geq 0$, where $\alpha_{n}$ are positive real numbers. Hence, $\gamma\left(\lambda_{n}\right)=0$. Having the spectrum $\left\{\lambda_{n}\right\}_{n \geq 0}$ we can construct the characteristic function (see [3, Theorem 1.1.4]):

$$
\Delta(\lambda)=\pi\left(\lambda-\lambda_{0}\right) \prod_{n=1}^{\infty} \frac{\lambda_{n}-\lambda}{n^{2}},
$$

and using asymptotics (3) for the eigenvalues we get the estimate

$$
\|\Delta(\lambda)\| \geq C_{\delta}|\rho| \exp (|\tau| \pi), \quad \rho \in G_{\delta} .
$$

Then we introduce $f(\lambda)=\frac{\gamma(\lambda)}{\Delta(\lambda)}$ and follow the proof of necessity in Theorem 1 .

In the general case, condition 3 is essential and cannot be omitted, that is shown by the following example.

Example 1. Let $m=2, \lambda_{01} \neq \lambda_{02}, \lambda_{n 1}=\lambda_{n 2}=n^{2}, n \geq 1$,

$$
\alpha_{01}=\alpha_{02}=\left[\begin{array}{ll}
\frac{1}{\pi} & 0 \\
0 & 0
\end{array}\right], \quad \alpha_{n 1}=\alpha_{n 2}=\left[\begin{array}{cc}
\frac{2}{\pi} & 0 \\
0 & \frac{2}{\pi}
\end{array}\right], n \geq 1 .
$$

The data $\left\{\lambda_{n q}, \alpha_{n q}\right\}$ satisfy conditions 1-2 of Theorem 1. Let us show that they do not satisfy condition 3, and consequently, they cannot be spectral data of $L$. The relations $\gamma\left(\lambda_{n q}\right) \alpha_{n q}=0$, 
$n \geq 0, q=\overline{1, m}$ for this example can be rewritten in the form $\gamma(\lambda)=\left[\gamma_{1}(\lambda), \gamma_{2}(\lambda)\right], \gamma_{1}\left(\lambda_{01}\right)=$ $\gamma_{1}\left(\lambda_{02}\right)=\gamma_{1}\left(n^{2}\right)=0, \gamma_{2}\left(n^{2}\right)=0, n \geq 1$. Clearly, if we put $\gamma_{1}(\lambda)=0, \gamma_{2}(\lambda)=\frac{\sin \rho \pi}{\rho}$, we arrive at a contradiction with condition 3 .

Below we investigate condition 3 in some special cases.

Example 2 (full multiplicities). Let $\lambda_{n 1}=\lambda_{n 2}=\ldots=\lambda_{n m}=: \lambda_{n}$ for all $n \geq 0$. Then rank $_{n q}=$ $m$, and each of the linear systems $\gamma\left(\lambda_{n q}\right) \alpha_{n q}=0$ has the unique solution $\gamma\left(\lambda_{n}\right)=0$. We get the situation similar to the scalar case, because in view of asymptotics (3), $\left\{\lambda_{n}\right\}_{n \geq 0}$ can be treated as eigenvalues of some scalar problem. Therefore, condition 3 holds automatically.

We will say that the relations $\gamma\left(\lambda_{n q}\right) \alpha_{n q}=0, q=\overline{1, m}$ are separated for some fixed $n$, if they yield $\gamma_{q}\left(\lambda_{n q}\right)=0$ for all $q=\overline{1, m}$. For example, they are separated in the case of full multiplicities, or when the matrices $\alpha_{n q}$ have a proper diagonal form.

Example 3. Let the relations $\gamma\left(\lambda_{n q}\right) \alpha_{n q}=0$ be separated for all $n>n_{0}$. Then each component $\gamma_{q}(\lambda)$ has zeros $\left\{\lambda_{n q}\right\}_{n>n_{0}}$. If $\gamma(\lambda)$ is the function from condition 3, each $\gamma_{q}(\lambda)$ cannot have more than $n_{0}$ additional zeros (counting with their multiplicities). Otherwise we consider its zeros as the eigenvalues of a scalar problem and prove that $\gamma_{q}(\lambda) \equiv 0$.

If $\gamma(\lambda)$ is entire, and $\gamma(\lambda)=O(\exp (|\operatorname{Im} \sqrt{\lambda}| \pi)), \quad|\lambda| \rightarrow \infty$, its order is not greater than $1 / 2$. Therefore, by Hadamard's factorization theorem $\gamma_{q}(\lambda)$ can be presented in the form

$$
\gamma_{q}(\lambda)=\left(C_{q 0}+C_{q 1} \lambda+C_{q 2} \lambda^{2}+\ldots+C_{q, n_{0}} \lambda^{n_{0}}\right) P_{q}(\lambda), \quad P_{q}(\lambda)=\prod_{n>n_{0}}\left(1-\frac{\lambda}{\lambda_{n q}}\right) .
$$

We substitute this factorization into $\gamma\left(\lambda_{n q}\right) \alpha_{n q}=0, n \leq n_{0}, q=\overline{1, m}$, and obtain the system of linear equations with respect to $C_{q 0}, C_{q 1}, \ldots, C_{q n_{0}}, q=\overline{1, m}$.

More precisely, let $\lambda_{1}, \ldots, \lambda_{N}$ be the first $N=\left(n_{0}+1\right) m$ eigenvalues, and let $\alpha_{1}, \ldots, \alpha_{N}$ be the corresponding residue-matrices. For each $j=\overline{1, N}$, we choose a non-zero column $v_{j}$ of $\alpha_{j}$. In case of a group of multiple values among $\lambda_{j}, j=\overline{1, N}$, they have a common matrix $\alpha_{j}$, and its rank equals their multiplicity, and we choose linearly independent columns. Consider $N \times N$ matrix $P$ with the columns

$$
\left[v_{j 1} P_{1}\left(\lambda_{j}\right), v_{j 1} \lambda_{j} P_{1}\left(\lambda_{j}\right), \ldots, v_{j 1} \lambda_{j}^{n_{0}} P_{1}\left(\lambda_{j}\right), \ldots, v_{j m} P_{m}\left(\lambda_{j}\right), v_{j m} \lambda_{j} P_{m}\left(\lambda_{j}\right), \ldots, v_{j m} \lambda_{j}^{n_{0}} P_{m}\left(\lambda_{j}\right)\right],
$$

$j=\overline{1, N}$. Clearly, that the condition $\gamma\left(\lambda_{n q}\right) \alpha_{n q}=0, n \leq n_{0}, q=\overline{1, m}$ is equivalent to the linear system with the matrix $P$. Each solution of this system corresponds to $\gamma(\lambda)$, satisfying condition 3 of Theorem 1 . Thus, the condition 3 is fulfilled iff the determinant of $P$ is not zero. 


\section{Solution of Inverse Problem 1}

3.1. Let the spectral data $\Lambda$ of the boundary value problem $L \in A(\omega), \omega \in D$, be given.

Denote

$$
D(x, \lambda, \mu)=\frac{\left\langle\varphi^{*}(x, \bar{\mu}), \varphi(x, \lambda)\right\rangle}{\lambda-\mu}=\int_{0}^{x} \varphi^{*}(t, \bar{\mu}) \varphi(x, \lambda) d t .
$$

We choose an arbitrary model boundary value problem $\tilde{L}=L(\tilde{Q}(x), \tilde{h}, \tilde{H}) \in A(\omega)$ (for example, one can take $\left.\tilde{Q}(x)=\frac{2}{\pi} \omega, \tilde{h}=0_{m}, \tilde{H}=0_{m}\right)$. We agree that if a certain symbol $\gamma$ denotes an object related to $L$, then the corresponding symbol $\tilde{\gamma}$ with tilde denotes the analogous object related to $\tilde{L}$. Put

$$
\xi_{n}=\sum_{q=1}^{m}\left|\rho_{n q}-\tilde{\rho}_{n q}\right|+\sum_{s=1}^{p} \sum_{q=m_{s}}^{m_{s+1}-1}\left|\rho_{n q}-\rho_{n m_{s}}\right|+\sum_{s=1}^{p} \sum_{q=m_{s}}^{m_{s+1}-1}\left|\tilde{\rho}_{n q}-\tilde{\rho}_{n m_{s}}\right|+\sum_{s=1}^{p}\left\|\alpha_{n}^{(s)}-\tilde{\alpha}_{n}^{(s)}\right\| .
$$

According to Lemmas 1 and 2,

$$
\Omega:=\left(\sum_{n=0}^{\infty}\left((n+1) \xi_{n}\right)^{2}\right)^{1 / 2}<\infty, \quad \sum_{n=0}^{\infty} \xi_{n}<\infty .
$$

Denote

$$
\begin{aligned}
& \lambda_{n q 0}=\lambda_{n q}, \quad \lambda_{n q 1}=\tilde{\lambda}_{n q}, \quad \rho_{n q 0}=\rho_{n q}, \quad \rho_{n q 1}=\tilde{\rho}_{n q}, \quad \alpha_{n q 0}^{\prime}=\alpha_{n q}^{\prime}, \quad \alpha_{n q 1}^{\prime}=\tilde{\alpha}_{n q}^{\prime}, \\
& \varphi_{n q i}(x)=\varphi\left(x, \lambda_{n q i}\right), \quad \tilde{\varphi}_{n q i}(x)=\tilde{\varphi}\left(x, \lambda_{n q i}\right), \\
& F_{k l j, n q i}(x)=\alpha_{k l j}^{\prime} D\left(x, \lambda_{n q i}, \lambda_{k l j}\right), \quad \tilde{F}_{k l j, n q i}(x)=\alpha_{k l j}^{\prime} \tilde{D}\left(x, \lambda_{n q i}, \lambda_{k l j}\right), \\
& n, k \geq 0, \quad q, l=\overline{1, m}, \quad i, j=0,1 .
\end{aligned}
$$

By the standard way (see [3, Lemma 1.6.2]), using Schwarz's lemma, we get

Lemma 6. The following estimates are valid for $x \in[0, \pi], n, k \geq 0, r, s=\overline{1, m}, m_{r}<q<m_{r+1}$, $m_{s}<l<m_{s+1}, i, j=0,1$ :

$$
\begin{aligned}
& \left\|\varphi_{n q i}(x)\right\| \leq C, \quad\left\|\varphi_{n m_{r} 0}(x)-\varphi_{n m_{r} 1}(x)\right\| \leq C \xi_{n}, \\
& \left\|\varphi_{n q i}(x)-\varphi_{n m_{r} i}(x)\right\| \leq C \xi_{n}, \quad\left\|F_{k l j, n q i}(x)\right\| \leq \frac{C}{|n-k|+1}, \\
& \left\|\sum_{l=m_{s}}^{m_{s+1}-1}\left(F_{k l 0, n m_{r} 1}(x)-F_{k l 1, n m_{r} 1}(x)\right)\right\| \leq \frac{C \xi_{k}}{|n-k|+1}, \\
& \left\|F_{k l j, n q i}(x)-F_{k l j, n m_{r} i}(x)\right\|,\left\|F_{k l j, n m_{r} 0}(x)-F_{k l j, n m_{r} 1}(x)\right\| \leq \frac{C \xi_{n}}{|n-k|+1}, \\
& \left\|\sum_{l=m_{s}}^{m_{s+1}-1}\left(F_{k l 0, n q i}(x)-F_{k l 0, n m_{r} i}(x)-F_{k l 1, n q i}(x)+F_{k l 1, n m_{r} i}(x)\right)\right\| \leq \frac{C \xi_{n} \xi_{k}}{|n-k|+1}, \\
& \left\|\sum_{l=m_{s}}^{m_{s+1}-1}\left(F_{k l 0, n m_{r} 0}(x)-F_{k l 0, n m_{r} 1}(x)-F_{k l 1, n m_{r} 0}(x)+F_{k l 1, n m_{r} 1}(x)\right)\right\| \leq \frac{C \xi_{n} \xi_{k}}{|n-k|+1} .
\end{aligned}
$$

The analogous estimates are also valid for $\tilde{\varphi}_{n q i}(x), \tilde{F}_{k l j, n q i}(x)$. 
The lemma similar to the following one has been proved in [7] by the contour integral method.

\section{Lemma 7. The following relations hold}

$$
\begin{array}{r}
\tilde{\varphi}(x, \lambda)=\varphi(x, \lambda)+\sum_{k=0}^{\infty} \sum_{l=1}^{m}\left(\varphi_{k l 0}(x) \alpha_{k l 0}^{\prime} \tilde{D}\left(x, \lambda, \lambda_{k l 0}\right)-\varphi_{k l 1}(x) \alpha_{k l 1}^{\prime} \tilde{D}\left(x, \lambda, \lambda_{k l 1}\right)\right) \\
\tilde{D}(x, \lambda, \mu)-D(x, \lambda, \mu)=\sum_{k=0}^{\infty} \sum_{l=1}^{m}\left(D\left(x, \lambda_{k l 0}, \mu\right) \tilde{D}\left(x, \lambda, \lambda_{k l 0}\right)-D\left(x, \lambda_{k l 1}, \mu\right) \tilde{D}\left(x, \lambda, \lambda_{k l 1}\right) .\right.
\end{array}
$$

Both series converge absolutely and uniformly with respect to $x \in[0, \pi]$ and $\lambda, \mu$ on compact sets.

Analogously one can obtain the following relation

$$
\tilde{\Phi}(x, \lambda)=\Phi(x, \lambda)+\sum_{k=0}^{\infty} \sum_{l=1}^{m} \sum_{j=0}^{1}(-1)^{j} \varphi_{k l j}(x) \alpha_{k l j}^{\prime} \frac{\left\langle\tilde{\varphi}_{k l j}^{*}(x), \tilde{\Phi}(x, \lambda)\right\rangle}{\lambda-\lambda_{k l j}} .
$$

It follows from Lemma 7 that

$$
\begin{aligned}
\tilde{\varphi}_{n q i}(x) & =\varphi_{n q i}(x)+\sum_{k=0}^{\infty} \sum_{l=1}^{m}\left(\varphi_{k l 0} \tilde{F}_{k l 0, n q i}(x)-\varphi_{k l 1} \tilde{F}_{k l 1, n q i}(x)\right), \\
\tilde{F}_{\eta p \omega, n q i}(x)-F_{\eta p \omega, n q i}(x) & =\sum_{k=0}^{\infty} \sum_{l=1}^{m}\left(F_{\eta p \omega, k l 0}(x) \tilde{F}_{k l 0, n q i}(x)-F_{\eta p \omega, k l 1}(x) \tilde{F}_{k l 1, n q i}(x)\right)
\end{aligned}
$$

for $n, \eta \geq 0, q, p=\overline{1, m}, i, \omega=0,1$.

Denote

$$
\varepsilon_{0}(x)=\sum_{(k, l, j) \in V}(-1)^{j} \varphi_{k l j}(x) \alpha_{k l j}^{\prime} \tilde{\varphi}_{k l j}^{*}(x), \quad \varepsilon(x)=-2 \varepsilon_{0}^{\prime}(x) .
$$

Using (5) and Lemma 6 one can easily check that the series in (17) converges absolutely and uniformly on $[0, \pi]$, and the function $\varepsilon_{0}(x)$ is absolutely continuous, and the components of $\varepsilon(x)$ belong to $L_{2}(0, \pi)$.

Lemma 8. The following relations hold

$$
Q(x)=\tilde{Q}(x)+\varepsilon(x), \quad h=\tilde{h}-\varepsilon_{0}(0), \quad H=\tilde{H}+\varepsilon_{0}(\pi),
$$

Proof. Differentiating (13) twice with respect to $x$ and using (12) and (17) we get

$$
\begin{aligned}
\tilde{\varphi}^{\prime}(x, \lambda)-\varepsilon_{0}(x) \tilde{\varphi}(x, \lambda) & =\varphi^{\prime}(x, \lambda)+\sum_{k=0}^{\infty} \sum_{l=1}^{m} \sum_{j=0}^{1}(-1)^{j} \varphi_{k l j}^{\prime}(x) \alpha_{k l j}^{\prime} \tilde{D}\left(x, \lambda, \lambda_{k l j}\right) \\
\tilde{\varphi}^{\prime \prime}(x, \lambda) & =\varphi^{\prime \prime}(x, \lambda)+\sum_{k=0}^{\infty} \sum_{l=1}^{m} \sum_{j=0}^{1}(-1)^{j}\left[\varphi_{k l j}^{\prime \prime}(x) \alpha_{k l j}^{\prime} \tilde{D}\left(x, \lambda, \lambda_{k l j}\right)\right.
\end{aligned}
$$




$$
\left.+2 \varphi_{k l j}^{\prime}(x) \alpha_{k l j}^{\prime} \tilde{\varphi}_{k l j}^{*}(x) \tilde{\varphi}(x, \lambda)+\varphi_{k l j}(x) \alpha_{k l j}^{\prime}\left(\tilde{\varphi}_{k l j}^{*}(x) \tilde{\varphi}(x, \lambda)\right)^{\prime}\right]
$$

We replace here the second derivatives, using equation (1), and then replace $\varphi(x, \lambda)$, using (13). This yields

$$
\begin{aligned}
\tilde{Q}(x) \varphi(x, \lambda)= & Q(x) \tilde{\varphi}(x, \lambda)+\sum_{k=0}^{\infty} \sum_{l=1}^{m} \sum_{j=0}^{1}(-1)^{j}\left[\varphi_{k l j}(x) \alpha_{k l j}^{\prime}\left\langle\tilde{\varphi}_{k l j}^{*}(x), \tilde{\varphi}(x, \lambda)\right\rangle\right. \\
& \left.+2 \varphi_{k l j}^{\prime}(x) \alpha_{k l j}^{\prime} \tilde{\varphi}_{k l j}^{*}(x) \tilde{\varphi}(x, \lambda)+\varphi_{k l j}(x) \alpha_{k l j}^{\prime}\left(\tilde{\varphi}_{k l j}^{*}(x) \tilde{\varphi}(x, \lambda)\right)^{\prime}\right] .
\end{aligned}
$$

Cancelling terms with $\tilde{\varphi}^{\prime}(x, \lambda)$ we arrive at $Q(x)=\tilde{Q}(x)+\varepsilon(x)$.

Further,

$$
\tilde{\varphi}^{\prime}(0, \lambda)-\left(h+\varepsilon_{0}(0)\right) \tilde{\varphi}(0)=U(\varphi)+\sum_{k=0}^{\infty} \sum_{l=1}^{m} \sum_{j=0}^{1}(-1)^{j} U\left(\varphi_{k l j}\right) \alpha_{k l j}^{\prime} D\left(0, \lambda, \lambda_{k l j}\right)=0_{m}
$$

Since $\tilde{\varphi}(0, \lambda)=I_{m}, \tilde{\varphi}^{\prime}(0, \lambda)=\tilde{h}$, we obtain $h=\tilde{h}-\varepsilon_{0}(0)$.

Similarly, using (14) one can get

$$
\tilde{\Phi}^{\prime}(\pi, \lambda)+\left(H-\varepsilon_{0}(\pi)\right) \Phi(\pi, \lambda)=V(\Phi)+\sum_{k=0}^{\infty} \sum_{l=1}^{m} \sum_{j=0}^{1}(-1)^{j} V\left(\varphi_{k l j}\right) \alpha_{k l j}^{\prime} \frac{\left\langle\tilde{\varphi}_{k l j}^{*}(x), \tilde{\Phi}(x, \lambda)\right\rangle_{\mid x=\pi}}{\lambda-\lambda_{k l j}}
$$

For $j=0$ we have $V\left(\varphi_{k l 0}\right) \alpha_{k l 0}^{\prime}=0_{m}$. For $j=1$

$$
\left\langle\tilde{\varphi}_{k l 1}^{*}(x), \tilde{\Phi}(x, \lambda)\right\rangle_{\mid x=\pi}=\tilde{V}^{*}\left(\tilde{\varphi}_{k l 1}^{*}\right) \tilde{\Phi}(\pi, \lambda)-\tilde{\varphi}_{k l 1}^{*}(\pi) \tilde{V}(\tilde{\Phi}) .
$$

Recall that $V(\Phi)=0_{m}, \tilde{V}(\tilde{\Phi})=0_{m}$ and $\alpha_{k l 1}^{\prime} \tilde{V}^{*}\left(\tilde{\varphi}_{k l 1}^{*}\right)=0_{m}$. Consequently, we arrive at $\tilde{\Phi}^{\prime}(\pi, \lambda)+$ $\left(H-\varepsilon_{0}(\pi)\right) \Phi(\pi, \lambda)=0_{m}$. Together with $\tilde{V}(\tilde{\Phi})=0_{m}$ this yields $H=\tilde{H}+\varepsilon(\pi)$.

For each fixed $x \in[0, \pi]$, the relation (15) can be considered as a system of linear equations with respect to $\varphi_{n q i}(x), n \geq 0, q=\overline{1, m}, i=0,1$. But the series in (15) converges only "with brackets". Therefore, it is not convenient to use (15) as a main equation of the inverse problem. Below we will transfer (15) to a linear equation in a corresponding Banach space of sequences.

3.2. Denote $\chi_{n}:=\xi_{n}^{-1}$ for $\xi_{n} \neq 0$ and $\chi_{n}=0$ for $\xi_{n}=0$. Let $V$ be a set of indices $u=(n, q, i)$, $n \geq 0, q=\overline{1, m}, i=0,1$. For each fixed $x \in[0, \pi]$, we define the row-vector $\psi(x)=\left[\psi_{u}(x)\right]_{u \in V}$ 
and the matrix $R(x)=\left[R_{v, u}(x)\right]_{v, u \in V}, v=(k, l, j), u=(n, q, i)$, by the formulae

$$
\begin{gathered}
\psi_{n m_{s} 0}(x)=\chi_{n}\left(\varphi_{n m_{s} 0}(x)-\varphi_{n m_{s} 1}(x)\right), \quad \psi_{n m_{s} 1}(x)=\varphi_{n m_{s} 1}(x), \\
\psi_{n q i}(x)=\chi_{n}\left(\varphi_{n q i}(x)-\varphi_{n m_{s} i}(x)\right), \\
R_{k m_{s} 0, n m_{r} 0}(x)=\chi_{n} \xi_{k} \sum_{l=m_{s}}^{m_{s+1}-1}\left(F_{k l 0, n m_{r} 0}(x)-F_{k l 0, n m_{r} 1}(x)\right), \\
R_{k m_{s} 0, n m_{r} 1}(x)=\xi_{k} \sum_{l=m_{s}}^{m_{s+1}-1} F_{k l 0, n m_{r} 1}(x), \\
R_{k m_{s} 0, n q i}(x)=\chi_{n} \xi_{k} \sum_{l=m_{s}}^{m_{s+1}-1}\left(F_{k l 0, n q i}(x)-F_{k l 0, n m_{r} i}(x)\right), \\
R_{k l j, n m_{r} 0}(x)=(-1)^{j} \chi_{n} \xi_{k}\left(F_{k l j, n m_{r} 0}(x)-F_{k l j, n m_{r} 1}(x)\right), \\
R_{k l j, n m_{r} 1}(x)=(-1)^{j} \xi_{k} F_{k l j, m_{r} 1}(x), \\
R_{k l j, n q i}(x)=(-1)^{j} \chi_{n} \xi_{k}\left(F_{k l j, n q i}(x)-F_{k l j, n m_{r} i}(x)\right), \\
R_{k m_{s} 1, n m_{r} 0}(x)=\chi_{n} \sum_{l=m_{s}}^{m_{s+1}-1}\left(F_{k l 0, n m_{r} 0}(x)-F_{k l 0, n m_{r} 1}(x)\right. \\
\left.-F_{k l 1, n m_{r} 0}(x)+F_{k l 1, n m_{r} 1}(x)\right), \\
R_{k m_{s} 1, n q i}(x)=\chi_{n} \sum_{l=m_{s}}^{m_{s+1}-1}\left(F_{k l 0, n q i}(x)-F_{k l 0, n m_{r} i}(x)-F_{k l 1, n q i}(x)+F_{k l 1, n m_{r} i}(x)\right), \\
R_{k m_{s} 1, n m_{r} 1}(x)=\sum_{l=m_{s}}^{m_{s+1}-1}\left(F_{k l 0, n m_{r} 1}(x)-F_{k l 1, n m_{r} 1}(x)\right), \\
n, k \geq 0, \quad r, s=\overline{1, p}, \quad m_{s}<l<m_{s+1}, \quad m_{r}<q<m_{r+1} .
\end{gathered}
$$

Analogously we define $\tilde{\psi}(x), \tilde{R}(x)$ by replacing in the previous definitions $\varphi_{n q i}(x)$ by $\tilde{\varphi}_{n q i}(x)$ and $F_{k l j, n q i}(x)$ by $\tilde{F}_{k l j, n q i}(x)$.

We will also use a shorter notation. Consider the row vectors with matrix components

$$
\begin{aligned}
\varphi_{n}(x) & =\left[\varphi_{n 10}(x), \varphi_{n 11}(x), \varphi_{n 20}(x), \varphi_{n 21}(x), \ldots, \varphi_{n m 0}(x), \varphi_{n m 1}(x)\right], \\
\psi_{n}(x) & =\left[\psi_{n 10}(x), \psi_{n 11}(x), \psi_{n 20}(x), \psi_{n 21}(x), \ldots, \psi_{n m 0}(x), \psi_{n m 1}(x)\right], \quad n \geq 0,
\end{aligned}
$$

and defined analogously $2 m \times 2 m$ matrices $F_{k, n}^{-}(x), R_{k, n}(x), n, k \geq 0, F_{k l j, n q i}^{-}(x)=(-1)^{j} F_{k l j, n q i}(x)$. Then definitions (19) of $\psi_{n q i}(x)$ and $R_{k l j, n q i}(x)$ can be rewritten in the form

$$
\psi_{n}=\varphi_{n} X_{n}, \quad R_{k, n}=X_{k}^{-1} F_{k, n}^{-} X_{n}, \quad n, k \geq 0
$$

where $X_{n}$ is a $2 m \times 2 m$ matrix with components determined from (19). Analogously we define $\tilde{\varphi}_{n}(x), \tilde{\psi}_{n}(x)$ and $\tilde{F}_{k, n}^{-}(x), \tilde{R}_{k, n}(x)$. Now we can rewrite (15) and (16) in the form

$$
\begin{aligned}
\tilde{\varphi}_{n} & =\varphi_{n}+\sum_{k=0}^{\infty} \varphi_{k} \tilde{F}_{k, n}^{-}, \quad n \geq 0, \\
\tilde{F}_{\eta, n}^{-}-F_{\eta, n}^{-} & =\sum_{k=0}^{\infty} F_{\eta, k}^{-} \tilde{F}_{k, n}^{-}
\end{aligned}
$$


By virtue of Lemma 6

$$
\begin{aligned}
\left\|\psi_{n q i}(x)\right\|,\left\|\tilde{\psi}_{n q i}(x)\right\| & \leq C, \\
\left\|R_{k l j, n q i}(x)\right\|,\left\|\tilde{R}_{k l j, n q i}(x)\right\| & \leq \frac{C \xi_{k}}{|n-k|+1},
\end{aligned}
$$

where $C$ does not depend on $x, n, q, i, k, l, j$

Let $a_{u}, u \in V$, be $m \times m$ matrices. Consider the Banach space $B$ of bounded sequences $a=\left[a_{u}\right]_{u \in V}$ with the norm $\|a\|_{B}=\sup _{u \in V}\left\|a_{u}\right\|$. It follows from (23) that for each fixed $x \in[0, \pi]$, the operators $I+\tilde{R}(x)$ and $I-R$ (here I is the identity operator), acting from $B$ to $B$, are linear bounded operators.

Theorem 2. For each fixed $x \in[0, \pi]$, the vector $\psi(x) \in B$ satisfies the equation

$$
\tilde{\psi}(x)=\psi(x)(I+\tilde{R}(x))
$$

in Banach space B. Moreover, the operator $I+\tilde{R}(x)$ has a bounded inverse operator, i. e. equation (24) is uniquely solvable.

Proof. Using (20) we get

$$
\varphi_{n}=\psi_{n} X_{n}^{-1}, \quad F_{k, n}^{-}=X_{k} R_{k, n} X_{n}^{-1},
$$

Substituting these relations into (21), we derive

$$
\tilde{\psi}_{n} X_{n}^{-1}=\psi_{n} X_{n}^{-1}+\sum_{k=0}^{\infty} \psi_{k} X_{k}^{-1} X_{k} \tilde{R}_{k, n} X_{n}^{-1}=\psi_{n} X_{n}^{-1}+\sum_{k=0}^{\infty} \psi_{k} \tilde{R}_{k, n} X_{n}^{-1} \quad n \geq 0 .
$$

Multiplying the result by $X_{n}$, we arrive at (24).

Similarly we get from (22) that

$$
\tilde{R}_{\eta, n}-R_{\eta, n}=\sum_{k=0}^{\infty} R_{\eta, k} \tilde{R}_{k, n}
$$

This yields $\tilde{R}(x)-R(x)-R(x) \tilde{R}(x)=0$, i. e. $(I-R(x))(I+\tilde{R}(x))=I$. Symmetrically, one gets $(I+\tilde{R}(x))(I-R(x))=I$. Hence the operator $(I+\tilde{R}(x))^{-1}$ exists, and it is a linear bounded operator.

Equation (24) is called the main equation of the inverse problem. Solving (24) we find the vector $\psi(x)$, and consequently, the functions $\varphi_{n q i}(x)$ by formulae

$$
\begin{gathered}
\varphi_{n m_{s} 1}(x)=\psi_{n m_{s} 1}(x), \quad \varphi_{n m_{s} 0}(x)=\varphi_{n m_{s} 1}(x)+\xi_{n} \psi_{n m_{s} 0}(x), \\
\varphi_{n q i}(x)=\varphi_{n m_{s} i}(x)+\xi_{n} \psi_{n q i}(x), \\
n \geq 0, s=\overline{1, p}, m_{s}<q<m_{s+1}, i=0,1 .
\end{gathered}
$$


Then we construct the potential $Q(x)$ and the coefficients of the boundary conditions $h$ and $H$ via (18). Thus, we obtain the following algorithm for the solution of Inverse Problem 1.

Algorithm 1. Given the data $\Lambda$.

(1) Choose $\tilde{L} \in A(\omega)$, and calculate $\tilde{\psi}(x)$ and $\tilde{R}(x)$.

(2) Find $\psi(x)$ by solving equation (24), and calculate $\varphi_{n q i}(x)$.

(3) Construct $Q(x), h$ and $H$ by (18).

\section{Sufficiency}

4.1. Let data $\left\{\lambda_{n q}, \alpha_{n q}\right\}_{n \geq 0, q=\overline{1, m}} \in$ Sp satisfying the conditions of Theorem 1 be given. Choose $\tilde{L} \in A(\omega)$, construct $\tilde{\psi}(x), \tilde{R}(x)$, and consider the equation (24).

Lemma 9. For each fixed $x \in[0, \pi]$, the operator $I+\tilde{R}(x)$, acting from $B$ to $B$, has a bounded inverse operator, and the main equation (24) has a unique solution $\psi(x) \in B$.

Proof. It is sufficient to prove that the homogeneous equation

$$
\beta(x)(I+\tilde{R}(x))=0
$$

where $\beta(x)=\left[\beta_{u}(x)\right]_{u \in V}, \beta_{u}(x)$ are $m \times m$ matrices, has only the zero solution. Let $\beta(x) \in B$ be a solution of (26), i. e.

$$
\beta_{n q i}(x)+\sum_{(k, l, j) \in V} \beta_{k l j}(x) \tilde{R}_{k l j, n q i}(x)=0_{m}
$$

Denote

$$
\begin{aligned}
\gamma_{n m_{s} 1}(x)= & \beta_{n m_{s} 1}(x), \quad \gamma_{n m_{s} 0}(x)=\gamma_{n m_{s} 1}(x)+\xi_{n} \beta_{n m_{s} 0}(x), \\
\gamma_{n q i}(x)= & \gamma_{n m_{s} i}(x)+\xi_{n} \beta_{n q i}(x), \\
& n \geq 0, s=\overline{1, p}, m_{s}<q<m_{s+1}, i=0,1 .
\end{aligned}
$$

Then $\gamma_{n q i}(x)$ satisfy the relations

$$
\gamma_{n q i}(x)+\sum_{k=0}^{\infty} \sum_{l=1}^{m}\left(\gamma_{k l 0}(x) \tilde{F}_{k l 0, n q i}(x)-\gamma_{k l 1}(x) \tilde{F}_{k l 1, n q i}(x)\right)=0_{m}, \quad n \geq 0,
$$

and the following estimates are valid

$$
\begin{gathered}
\left\|\gamma_{n q i}(x)\right\| \leq C(x), \quad n \geq 0, \quad q=\overline{1, m}, \\
\left\|\gamma_{n m_{s} 0}(x)-\gamma_{n m_{s} 1}(x)\right\|,\left\|\gamma_{n q i}(x)-\gamma_{n m_{s} i}(x)\right\| \leq C(x) \xi_{n}, \\
s=\overline{1, p}, m_{s}<q<m_{s+1} .
\end{gathered}
$$


Construct the matrix-functions $\gamma(x, \lambda), \Gamma(x, \lambda)$ and $B(x, \lambda)$ by the formulas

$$
\begin{aligned}
& \gamma(x, \lambda)=-\sum_{k=0}^{\infty} \sum_{l=1}^{m}\left[\gamma_{k l 0}(x) \alpha_{k l 0}^{\prime} \frac{\left\langle\tilde{\varphi}_{k l 0}^{*}(x), \tilde{\varphi}(x, \lambda)\right\rangle}{\lambda-\lambda_{k l 0}}-\gamma_{k l 1}(x) \alpha_{k l 1}^{\prime} \frac{\left\langle\tilde{\varphi}_{k l 1}^{*}(x), \tilde{\varphi}(x, \lambda)\right\rangle}{\lambda-\lambda_{k l 1}}\right], \\
& \Gamma(x, \lambda)=-\sum_{k=0}^{\infty} \sum_{l=1}^{m}\left[\gamma_{k l 0}(x) \alpha_{k l 0}^{\prime} \frac{\left\langle\tilde{\varphi}_{k l 0}^{*}(x), \tilde{\Phi}(x, \lambda)\right\rangle}{\lambda-\lambda_{k l 0}}-\gamma_{k l 1}(x) \alpha_{k l 1}^{\prime} \frac{\left\langle\tilde{\varphi}_{k l 1}^{*}(x), \tilde{\Phi}(x, \lambda)\right\rangle}{\lambda-\lambda_{k l 1}}\right], \\
& B(x, \lambda)=\gamma^{*}(x, \bar{\lambda}) \Gamma(x, \lambda) .
\end{aligned}
$$

In view of (12), the matrix-function $\gamma(x, \lambda)$ is entire in $\lambda$ for each fixed $x$. The functions $\Gamma(x, \lambda)$ and $B(x, \lambda)$ are meromorphic in $\lambda$ with simple poles $\lambda_{n q i}$. According to (29), $\gamma\left(x, \lambda_{n q i}\right)=\gamma_{n q i}(x)$. We calculate residues of $B(x, \lambda)$ (for simplicity we assume that $\left\{\lambda_{n q 0}\right\} \cap$ $\left.\left\{\lambda_{n q 1}\right\}=\varnothing\right):$

$$
\operatorname{Res}_{\lambda=\lambda_{n q 0}} B(x, \lambda)=\gamma^{*}\left(x, \lambda_{n q 0}\right) \gamma\left(x, \lambda_{n q 0}\right) \alpha_{n q 0}, \quad \operatorname{Res}_{\lambda=\lambda_{n q 1}} B(x, \lambda)=0_{m} .
$$

Consider the integral

$$
I_{N}(x)=\frac{1}{2 \pi i} \int_{\Gamma_{N}} B(x, \lambda) d \lambda,
$$

where $\Gamma_{N}=\left\{\lambda:|\lambda|=(N+1 / 2)^{2}\right\}$. Let us show that for each fixed $x \in[0, \pi]$

$$
\lim _{N \rightarrow \infty} I_{N}(x)=0_{m}
$$

Indeed, it follows from (12) and (29) that

$$
\begin{aligned}
-\gamma(x, \lambda)= & \sum_{k=0}^{\infty} \sum_{s=1}^{p} \sum_{l=m_{s}}^{m_{s+1}-1}\left[\gamma_{k l 0}(x) \alpha_{k l 0}^{\prime} \tilde{D}\left(x, \lambda, \lambda_{k l 0}\right)-\gamma_{k l 1}(x) \alpha_{k l 1}^{\prime} \tilde{D}\left(x, \lambda, \lambda_{k l 1}\right)\right] \\
= & \sum_{k=0}^{\infty} \sum_{s=1}^{p}\left[\left(\gamma_{k m_{s} 0}(x)-\gamma_{k m_{s} 1}(x)\right) \sum_{l=m_{s}}^{m_{s+1}-1} \alpha_{k l 0}^{\prime} \tilde{D}\left(x, \lambda, \lambda_{k l 0}\right)+\gamma_{k m_{s} 1}(x) \alpha_{k}^{(s)}\left(\tilde{D}\left(x, \lambda, \lambda_{k m_{s} 0}\right)\right.\right. \\
& \left.-\tilde{D}\left(x, \lambda, \lambda_{k m_{s} 1}\right)\right)+\gamma_{k m_{s} 1}(x)\left(\alpha_{k}^{(s)}-\tilde{\alpha}_{k}^{(s)}\right) \tilde{D}\left(x, \lambda, \lambda_{k m_{s} 1}\right) \\
& +\gamma_{k m_{s} 1}(x) \sum_{l=m_{s}}^{m_{s+1}-1} \sum_{j=0}^{1} \alpha_{k l j}^{\prime}\left(\tilde{D}\left(x, \lambda, \lambda_{k l j}\right)-\tilde{D}\left(x, \lambda, \lambda_{k m_{s} j}\right)\right) \\
& \left.+\sum_{l=m_{s}}^{m_{s+1}-1} \sum_{j=0}^{1}\left(\gamma_{k l j}(x)-\gamma_{k m_{s} j}(x)\right) \alpha_{k l j}^{\prime} \tilde{D}\left(x, \lambda, \lambda_{k l j}\right)\right] .
\end{aligned}
$$

By virtue of Lemma 6, (5) and (28), we get

$$
\|\gamma(x, \lambda)\| \leq C(x) \exp (|\tau| x) \sum_{k=0}^{\infty} \frac{\xi_{k}}{|\rho-k|+1}, \quad \operatorname{Re} \rho \geq 0 .
$$

Similarly, using (30) we obtain for sufficiently large $\rho^{*}>0$ :

$$
\|\Gamma(x, \lambda)\| \leq \frac{C(x)}{|p|} \exp (-|\tau| x) \sum_{k=0}^{\infty} \frac{\xi_{k}}{|\rho-k|+1}, \operatorname{Re} \rho \geq 0,|\rho| \geq \rho^{*}, \rho \in G_{\delta} .
$$


Then

$$
\|B(x, \lambda)\| \leq \frac{C(x)}{|\rho|}\left(\sum_{k=0}^{\infty} \frac{\xi_{k}}{|\rho-k|+1}\right)^{2} \leq \frac{C(x)}{|\rho|^{3}}, \quad \lambda \in \Gamma_{N} .
$$

This estimate yields $\lim _{N \rightarrow \infty} I_{N}(x)=0_{m}$.

On the other hand, calculating the integral $I_{N}(x)$ by the residue theorem, we arrive at

$$
\sum_{k=0}^{\infty} \sum_{q=1}^{m} \gamma_{k l 0}^{*}(x) \gamma_{k l 0}(x) \alpha_{k l 0}^{\prime}=0_{m}
$$

Since $\alpha_{k l 0}=\alpha_{k l 0}^{*} \geq 0$, we get

$$
\begin{aligned}
\gamma_{k l 0}^{*}(x) \gamma_{k l 0}(x) \alpha_{k l 0} & =0_{m}, \\
\gamma\left(x, \lambda_{k l 0}\right) \alpha_{k l 0} & =0_{m}, \quad k \geq 0, \quad l=\overline{1, m} .
\end{aligned}
$$

Since $\gamma(x, \lambda)$ is entire in $\lambda$, and

$$
\gamma(x, \lambda)=O(\exp (|\tau| x))
$$

for each fixed $x \in[0, \pi]$, according to condition 3 of Theorem 1 , we get $\gamma(x, \lambda) \equiv 0_{m}$. Therefore $\gamma_{n q i}(x)=0_{m}$ for all $n \geq 0, q=\overline{1, m}, i=0,1$, i. e. the homogeneous equation (26) has only the zero solution.

4.2. Further, we provide the general strategy of the proof of sufficiency in Theorem 1 . The proofs of Lemmas 10-12 are similar to ones described in [3, Sec. 1.6.2].

Let $\psi(x)=\left[\psi_{u}(x)\right]_{u \in V}$ be the solution of the main equation (24).

Lemma 10. For $n \geq 0, q=\overline{1, m}, i=0,1$, the following relations hold

$$
\begin{gathered}
\psi_{n q i}(x) \in C^{1}[0, \pi], \quad\left\|\psi_{n q i}^{(v)}\right\| \leq C(n+1)^{v}, \quad v=0,1 \quad x \in[0, \pi], \\
\left\|\psi_{n q i}(x)-\tilde{\psi}_{n q i}(x)\right\| \leq C \Omega \eta_{n}, \quad\left\|\psi_{n q i}^{\prime}(x)-\tilde{\psi}_{n q i}^{\prime}(x)\right\| \leq C \Omega, \quad x \in[0, \pi],
\end{gathered}
$$

where

$$
\eta_{n}:=\left(\sum_{k=0}^{\infty} \frac{1}{(k+1)^{2}(|n-k|+1)^{2}}\right) .
$$

Construct matrix-functions $\varphi_{n q i}(x)$ by formulae (25). By virtue of Lemma 10, we have

$$
\begin{gathered}
\left\|\varphi_{n q i}^{(v)}(x)\right\| \leq C(n+1)^{v}, \quad v=0,1, \\
\left\|\varphi_{n q i}(x)-\tilde{\varphi}_{n q i}(x)\right\| \leq C \Omega \eta_{n}, \quad\left\|\varphi_{n q i}^{\prime}(x)-\tilde{\varphi}_{n q i}^{\prime}(x)\right\| \leq C \Omega, \quad q=\overline{1, m}, \\
\left\|\varphi_{n m_{s} 0}(x)-\varphi_{n m_{s} 1}(x)\right\|,\left\|\varphi_{n q i}(x)-\varphi_{n m_{s} i}(x)\right\| \leq C \xi_{n}, \quad s=\overline{1, p}, m_{s}<q<m_{s+1} .
\end{gathered}
$$


Further, we construct the matrix-functions $\varphi(x, \lambda)$ and $\Phi(x, \lambda)$ by the formulas

$$
\begin{aligned}
& \varphi(x, \lambda)=\tilde{\varphi}(x, \lambda)-\sum_{(k, l, j) \in V}(-1)^{j} \varphi_{k l j}(x) \alpha_{k l j}^{\prime} \frac{\left\langle\tilde{\varphi}_{k l j}^{*}(x), \tilde{\varphi}(x, \lambda)\right\rangle}{\lambda-\lambda_{k l j}}, \\
& \Phi(x, \lambda)=\tilde{\Phi}(x, \lambda)-\sum_{(k, l, j) \in V}(-1)^{j} \varphi_{k l j}(x) \alpha_{k l j}^{\prime} \frac{\left\langle\tilde{\varphi}_{k l j}^{*}(x), \tilde{\Phi}(x, \lambda)\right\rangle}{\lambda-\lambda_{k l j}},
\end{aligned}
$$

and the boundary value problem $L(Q(x), h, H)$ via (18). Clearly, $\varphi\left(x, \lambda_{n q i}\right)=\varphi_{n q i}(x)$.

Using estimates (31) one can show that the components of $\varepsilon_{0}(x)$ are absolutely continuous and the components of $\varepsilon(x)$ belong to $L_{2}(0, \pi)$. Consequently, we get

Lemma 11. $Q_{j k}(x) \in L_{2}(0, \pi), j, k=\overline{1, m}$.

Lemma 12. The following relations hold

$$
\begin{aligned}
\ell \varphi_{n q i}(x) & =\lambda_{n q i} \varphi_{n q i}(x), \quad \ell \varphi(x, \lambda)=\lambda \varphi(x, \lambda), \quad \ell \Phi(x, \lambda)=\lambda \Phi(x, \lambda), \\
\varphi(0, \lambda) & =I_{m}, \quad \varphi^{\prime}(0, \lambda)=h, \quad U(\Phi)=I_{m}, \quad V(\Phi)=0_{m} .
\end{aligned}
$$

In order to finish the proof of Theorem 1 it remains to show that the given data $\left\{\lambda_{n q}, \alpha_{n q}\right\}$ coincide with the spectral data of the constructed boundary value problem $L(Q, h, H)$. In view of Lemma 12, the matrix-function $\Phi(x, \lambda)$ is the Weyl solution of $L$. Let us get the representation for the Weyl matrix:

$$
\begin{aligned}
M(\lambda)= & \Phi(0, \lambda)=\tilde{M}(\lambda)-\sum_{(k, l, j) \in V} \varphi_{k l j}(0) \alpha_{k l j}^{\prime} \frac{\left\langle\tilde{\varphi}_{k l j}^{*}(x), \tilde{\Phi}(x, \lambda)\right\rangle_{x=0}}{\lambda-\lambda_{k l j}} \tilde{M}(\lambda) \\
& +\sum_{k=0}^{\infty} \sum_{l=1}^{m}\left(\frac{\alpha_{k l 0}^{\prime}}{\lambda-\lambda_{k l 1}}-\frac{\alpha_{k l 1}^{\prime}}{\lambda-\lambda_{k l 1}}\right) .
\end{aligned}
$$

Using the equality (see [4])

$$
\tilde{M}(\lambda)=\sum_{k=0}^{\infty} \sum_{l=1}^{m} \frac{\alpha_{k l 1}^{\prime}}{\lambda-\lambda_{k l 1}}
$$

we arrive at

$$
M(\lambda)=\sum_{k=0}^{\infty} \sum_{l=1}^{m} \frac{\alpha_{k l 0}^{\prime}}{\lambda-\lambda_{k l 0}}
$$

Consequently, $\left\{\lambda_{k l 0}\right\}$ are simple poles of the Weyl matrix $M(\lambda)$, and $\left\{\alpha_{k l 0}\right\}$ are residues at the poles. Note that the multiplicities of the eigenvalues coincide with the numbers of equal values among $\left\{\lambda_{k l 0}\right\}$, because they both coincide with the ranks of $\left\{\alpha_{k l 0}\right\}$. Theorem 1 is proved. 


\section{Acknowledgement}

This research was supported in part by Grants 10-01-00099 and 10-01-92001-NSC of Russian Foundation for Basic Research and Taiwan National Science Council and by the Moebius Contest Foundation for Young Scientists.

\section{References}

[1] Marchenko V.A. Sturm-Liouville Operators and their Applications, Naukova Dumka, Kiev, 1977 (Russian); English transl., Birkhauser, 1986.

[2] Levitan B.M. Inverse Sturm-Liouville Problems, Nauka, Moscow, 1984 (Russian); English transl., VNU Sci.Press, Utrecht, 1987.

[3] Freiling G. and Yurko V.A. Inverse Sturm-Liouville Problems and their Applications. NOVA Science Publishers, New York, 2001.

[4] Yurko V.A. Inverse problems for matrix Sturm-Liouville operators, Russian J. Math. Phys. 13, no.1 (2006), 111-118.

[5] Carlson R. An inverse problem for the matrix Schrödinger equation, J. Math. Anal. Appl. 267 (2002), 564-575.

[6] Malamud M.M. Uniqueness of the matrix Sturm-Liouville equation given a part of the monodromy matrix, and Borg type results. Sturm-Liouville Theory, Birkhäuser, Basel, 2005, 237-270.

[7] Yurko V.A. Inverse problems for the matrix Sturm-Liouville equation on a finite interval, Inverse Problems, 22 (2006), 1139-1149.

[8] Chelkak D., Korotyaev E. Weyl-Titchmarsh functions of vector-valued Sturm-Liouville operators on the unit interval, J. Func. Anal. 257 (2009), 1546-1588.

[9] Mykytyuk Ya.V., Trush N.S. Inverse spectral problems for Sturm-Liouville operators with matrix-valued potentials, Inverse Problems, 26 (2010), 015009.

[10] Naimark M.A. Linear Differential Operators, 2nd ed., Nauka, Moscow, 1969; English transl. of 1st ed., Parts I,II, Ungar, New York, 1967, 1968.

Department of Mathematics, Saratov State University, Astrakhanskaya, 83, Saratov 410026, Russia.

E-mail: bondarenkonp@info.sgu.ru 